\title{
Design and Performance Analysis of a Space-Time Block Coding scheme for Single Band UWB
}

\author{
Fabien Héliot, Mohammad Ghavami, and Mohammad Reza Nakhai
}

\begin{abstract}
Ultra wide-band (UWB) systems have recently attracted much research interest owing to their appealing features in short-range mobile communications. These features include high data rates, low power consumption, multiple access communications, and precise positioning capabilities. Space-time coding techniques, such as the block coding scheme or the trellis coding scheme, are known to be simple and practical ways to increase both the spectral efficiency and the capacity in wireless communications. So far, few contributions have looked over multiple inputs multiple outputs UWB systems. In this paper, a method to adapt the space-time block coding technique to single band UWB signalling is proposed. A space-time block codec, based on orthogonal pulses to achieve spatial diversity and collect the multipath diversity is developed. A theoretical analysis is conducted to enlighten the performance enhancements provided by the proposed scheme compared to the classic single link scheme. Simulations support analysis, for various numbers of transmit and receive antennas, several types of channel scenarios, and different detection techniques. In a typical studied UWB environment, the results revealed that our proposed scheme improves the bit error rate performances compared to those of a single link scheme and it provides a strong immunity against timing jitter.
\end{abstract}

Index Terms-Ultra wide-band communication systems, impulse radio, space-time block coding, diversity, rake receiver, pulse position modulation

\section{INTRODUCTION}

Impulse radio (IR), also known as single band ultra wideband (UWB), is defined as a form of ultra-wide bandwidth signalling which is well designed for base-band asynchronous multiple access (MA), short distance-high data rate multimedia services, and tactical wireless communications [1]. Different approaches have been already investigated in order to deploy a UWB system. These include a multi-band carrierbased approach supported by multi-carrier spread spectrum and a single band approach implemented via pulse position modulation (PPM), pulse amplitude modulation, pulse shape modulation or a combination of them [2]. In addition, random time-hopping (TH) or direct sequence (DS) codes are used to allow secure multiple user transmissions [3]. The analysis presented hereafter, considers a UWB communication system based on the single band UWB approach.

Multiple-inputs-multiple-outputs systems are known to provide higher capacity and therefore better performances than single link (SL) systems in wireless communications by employing multiple transmit, and optionally, multiple receive antennas. Most popular of them, such as space-time block

F. Héliot, M. Ghavami and M. R. Nakhai are with the Centre for Telecommunications Research, King's College London, University of London, Division of Engineering, Strand, London WC2R 2LS, UK (e-mail: F.Heliot@Surrey.ac.uk). coding (STBC) [4], [5], space-time trellis coded modulation [6], and layered space-time architectures [7], can provide high data rates with a given transceiver complexity. With $3 \mathrm{G}$ systems and beyond requiring high data rates for applications such as multimedia, this particular area of research has gained a lot of interest.

A multi-antenna architecture for UWB systems was first introduced in [8], where an Alamouti based STBC scheme [4] with two transmit antennas and one receive antenna is used over a simple channel model. This work was then extended in [9] to include performance assessments over a narrow band indoor channel model. Both works exhibit performance improvements compared to the SL-IR scheme, in terms of bit error rate (BER). In [10], we combined a multi-antenna single band UWB STBC (STBC-IR) system with orthogonal pulses to enhance the data rate. In this paper, we modify the scheme introduced in [10] and we propose a different method from the one presented in [9] to achieve spatial diversity regardless of the number of transmit and receive antennas. We developed our scheme for both non-coherent reception (NCR) and coherent reception (CR). In the case of CR, a fractionally spaced (FS) rake receiver with $F_{g}$ fingers and a maximum ratio combining (MRC) detector are used to collect the multipath diversity. As a particular significance of our work, we investigate the performance analysis of our scheme over a realistic single band UWB channel model, i.e., the IEEE 802.15.3a UWB channel model, reported in [11]. We derive our scheme mathematically based on this channel model. Then, we analytically determine upper bounds on the maximum achievable performances in terms of BER. Next, we assess the theoretical reachable diversity gain of our scheme and we compare it via analysis to the one of any SL-IR scheme. Finally, we confirm our theoretical results numerically, for various transmission scenarios, through Monte Carlo simulations.

The rest of the paper is organized as follows, Section II briefly introduces the IR-MA model, with both DS-UWB and TH-UWB as multiple access techniques. It also presents a detailed analysis in terms of channel model, transceiver structure and detection methods for a standard single-inputsingle-output (SISO) IR system. In Section III, an overview of modified Hermitian pulses (MHPs) [2] is given. Then, a comprehensive derivation of our STBC-IR scheme is carried out for a general multi-antenna configuration. In Section IV, simulation parameters are presented and performances are assessed in terms of BER. Results from Monte Carlo simulations are plotted and compared with their theoretical counterparts. Finally, conclusions are drawn in Section V. 


\section{SYSTEM MODEL FOR SISO IR-MA}

\section{A. IR-MA signaling model}

This part of the paper briefly describes the IR-MA signalling model [3]. The transmitted signal $\omega_{u}(t)$, from the $u$ th user to any other user, can be described as a train of short pulses $w(t)$, where $T_{w}$ is the width of each pulse within the train. Each transmitted symbol is represented by several similar pulses. Each of this pulse is transmitted within a frame. The duration of a frame is denoted $T_{f}$, where $T_{f} \geq T_{w}$. The number of frames per transmitted symbol is $N_{f}$. Therefore, the duration of each symbol $T_{s}$ is such as $T_{s}=N_{f} T_{f}$. If $N_{u}$ users are accommodated applying $\mathrm{TH}$ codes, then the frame duration $T_{f}$ can expressed such as $T_{f}=N_{c} T_{c}+T_{g}$, where $N_{c}$ is the number of chips within the frame, $T_{c}$ is the chip duration and $T_{g}$ is a guard period for processing delay. Each frame contains only one pulse per user. Considering a single band $M$-ary PPM TH-UWB communication system, where packets of $N_{s}$ symbols are transmitted, the mathematical expression of the $u$ th user transmitted signal at any time $t$ is given by

$$
\begin{aligned}
\omega_{u}(t) & =\sqrt{\epsilon_{u}} \sum_{i=0}^{N_{s}-1} \sum_{k=0}^{N_{f}-1} w\left(t-\left(i N_{f}+k\right) T_{f},\right. \\
& \left.-C_{u}(k) T_{c}-T_{I_{u}(i)}\right)
\end{aligned}
$$

where $\epsilon_{u}$ is the $u$ th user transmitted symbol energy, $C_{u}(k)$ is a $N_{p}$ periodic TH pseudo-random code, such as $C_{u}(k) \in$ $\left\{0, N_{c}-1\right\}, I_{u}(i)$ represents the information bearing the $i$ th transmitted symbol sent by the $u$ th user, $I_{u}(i) \in\{0, M-$ $1\}$, and $T_{I_{u}(i)}$ is the delay related to $I_{u}(i)$. Considering a $M$-ary orthogonal PPM (OPPM), this delay can be expressed such as $T_{I_{u}(i)}=\left(I_{u}(i) / M\right) T_{s}$. This delay represents the pulse position shift in the set of all possible position shifts, according to the $M$-ary modulation. Otherwise, one can also combine DS codes with UWB system to accommodate several users. In that case, $C_{u}(k)$ is called the direct spread sequence coefficient, and $C_{u}(k)= \pm 1$. Considering a single band $M$-ary PPM DSUWB communication system, the $u$ th transmitted signal can be expressed such as

$\omega_{u}(t)=\sqrt{\epsilon_{u}} \sum_{i=0}^{N_{s}-1} \sum_{k=0}^{N_{f}-1} C_{u}(k) w\left(t-\left(i N_{f}+k\right) T_{f}-T_{I_{u}(i)}\right)$.

The number of users accommodated and the level of intersymbol interferences (ISI) can be tuned via the pulse repetition gain, which is equal to $10 \log _{10}\left(N_{f}\right)$ and the duty cycle gain, which is equal to $10 \log _{10}\left(\frac{T_{f}}{T_{w}}\right)$. A low repetition gain implies more ISI, multiple user collisions and synchronization problems. A high repetition gain implies a lower data rate. Considering the TH-UWB MA technique, a low duty cycle provides a good protection against catastrophic collisions in a multi-user environment [3].

\section{B. Single link Configuration}

A detailed analysis of a SISO single band UWB communication system is undertaken here. Several aspects of the system are studied in this Section such as the transceiver architecture, the propagation medium and different detection methods.
At any given time, a bit stream flows toward the input of the system. Each bit is mapped into a symbol via a $M$-level modulator. Then, each symbol is shaped into its corresponding waveform via a pulse shaper, depending on the type of modulation and the multiuser technique chosen. Assuming packets of $N_{s}$ symbols are encoded through a $M$ ary orthogonal pulse position modulator $\left(T_{f}=M T_{w}\right)$ and they are transmitted via a DS-UWB communication system, the expression of the $u$ th user transmitted signal per symbol $i$ at any time $t$ is given by

$\omega_{u}(t, i)=\sqrt{\frac{\epsilon_{u}}{N_{f}}} \sum_{k=0}^{N_{f}-1} C_{u}(k) w\left(t-\left(\left[i M+I_{u}(i)\right] N_{f}+k\right) T_{w}\right)$,

where $w(t)$ is a normalised to unit-energy pulse $\left(\int_{0}^{T_{f}} w^{2}(t)=1\right), \sqrt{\epsilon_{u} / N_{f}}$ is a normalization coefficient, and $i \in\left\{0, N_{s}-1\right\}$. The signal $\omega_{u}(t, i)$ will be distorted during its transmission. In a single band UWB typical environment (indoor environment), the Doppler spectrum is quasi-constant. Therefore, the propagation medium can be labelled as a very slow fading channel, and it can be modelled by a finite impulse response signal [11]

$$
h(t)=X \sum_{p=0}^{P-1} \sum_{q=0}^{Q-1} \alpha_{p, q} \delta\left(t-T_{p}-\tau_{p, q}\right)
$$

where $\alpha_{p, q}=\beta_{p, q} e^{j \theta_{p, q}}$ is the multipath gain of ray $q$ within the $p$ th cluster with $\beta_{p, q}$ being log-normally distributed and $\theta_{p, q}=0$ or $\pi, T_{p}$ is the arrival time of the first ray of the $p$ th cluster and $\tau_{p, q}$ is the delay of the $q$ th ray within the $p$ th cluster relative to the first ray arrival time of that cluster. $X$ models the log-normal shadowing, $X=10^{(Y / 20)}$, where $Y$ follows a normal distribution, with $0 \mathrm{~dB}$ mean value and $3 \mathrm{~dB}$ standard deviation. The model in (4) is the IEEE 802.15.3a UWB channel model [11] and it is based on the SalehValenzuela model [12]. Considering perfect synchronization, the $u$ th user received signal $r_{u}(t, i)$ per transmitted symbol is simply the convolution of $\omega_{u}(t, i)$ with the channel impulse response $h(t)$, plus an additive gaussian noise $n(t)$ with twosided power spectral density $N_{0} / 2$ as follows

$$
r_{u}(t, i)=X \sum_{p=0}^{P-1} \sum_{q=0}^{Q-1} \alpha_{p, q} \omega_{u}\left(t-T_{p}-\tau_{p, q}, i\right)+n(t) .
$$

At this stage, both NCR and CR techniques can be used to retrieve transmitted symbols within the received signal. A coherent receiver needs to estimate the channel parameters $\alpha_{p, q}, T_{p}, \tau_{p, q}$, for instance by applying the matched filter technique along with a FS-rake receiver and exploiting data aided (DA) channel estimation [13]. Each estimation of the signal $h(t)$ is denoted by $\widetilde{h}(t)$ and expressed as

$$
\widetilde{h}(t)=\widetilde{X} \sum_{f=0}^{F_{g}-1} \widetilde{\alpha}_{f} \delta\left(t-\widetilde{\tau}_{f}\right)
$$

where $F_{g}$ is the number of fingers of the FS-rake receiver, $\widetilde{X}$ is the estimation of the shadowing effects, $\widetilde{\alpha}_{f}$ is the $f$ th estimated multipath gain of the estimated channel impulse 
response and $\widetilde{\tau}_{f}$ is the estimated delay of the corresponding multipath gain. At the receiver side, the multiuser waveform of the desired user $u$ is generated according to

$\omega_{u}(t, i, m)=\sqrt{\frac{1}{N_{f}}} \sum_{k=0}^{N_{f}-1} C_{u}(k) w\left(t-\left([i M+m] N_{f}+k\right) T_{w}\right)$,

where $m \in\{0, M-1\}$. The convolution of $\omega_{u}(t, i, m)$ with $\widetilde{h}(t)$ produces an estimated template waveform $s_{\mathrm{CR} u}(t, i, m)$ per user, per transmitted symbol and per possible position as follows

$$
s_{\mathrm{CR} u}(t, i, m)=\widetilde{X} \sum_{f=0}^{F_{g}-1} \widetilde{\alpha}_{f} \omega_{u}\left(t-\widetilde{\tau}_{f}, i, m\right) .
$$

Next, the correlation between the received signal $r_{u}(t, i)$ and each possible $s_{\mathrm{CR} u}(t, i, m)$ is performed for each transmitted symbol over $T_{s}+\widetilde{\tau}_{F_{g}-1}$, where $T_{s}=M N_{f} T_{w}$ and $\widetilde{\tau}_{F_{g}-1}$ is the greatest delay of $\widetilde{h}(t)$. Thus, a decision statistic towards the decoding of the $i$ th transmitted symbol is provided by

$$
\Lambda_{u}(i, m)=\int_{(i M+m) N_{f} T_{w}}^{(i M+m+1) N_{f} T_{w}+\widetilde{\tau}_{F_{g}-1}} r_{u}(t, i) s_{\mathrm{CR} u}(t, i, m) d t .
$$

In the case of NCR, a known waveform is transmitted at the beginning of each new packet through the slow fading channel modelled according to [11]. The known received waveform $s_{\mathrm{NCR} u}(t)$ can be expressed as

$$
\begin{aligned}
s_{\mathrm{NCR} u}(t) & =X \sqrt{\frac{\epsilon_{u}}{N_{f}}} \sum_{k=0}^{N_{f}-1} \sum_{p=0}^{P-1} \sum_{q=0}^{Q-1} \alpha_{p, q} \\
& \times C_{u}(k) w\left(t-T_{p}-\tau_{p, q}-k T_{w}\right)+n^{\prime}(t)
\end{aligned},
$$

where $n^{\prime}(t)$ is an additive gaussian noise with two-sided power spectral density $N_{0} / 2$. Then, $M$ translated replicas of $s_{\mathrm{NCR} u}(t)$ for the $i$ th transmitted symbol are generated according to

$s_{\mathrm{NCR} u}(t, i, m)=\int_{\mathbb{R}} \delta\left(t-(i M+m) N_{f} T_{w}-\tau\right) s_{\mathrm{NCR} u}(\tau) d \tau$.

Next, the correlation between the received signal $r_{u}(t, i)$ and each possible $s_{\mathrm{NCR} u}(t, i, m)$ is performed for each transmitted symbol over $T_{s}$. A decision statistic towards the decoding of the $i$ th transmitted symbol is given by

$$
\Lambda_{u}(i, m)=\int_{(i M+m) N_{f} T_{w}}^{(i M+m+1) N_{f} T_{w}} r_{u}(t, i) s_{\mathrm{NCR} u}(t, i, m) d t .
$$

Eventually, we perform the maximum-likelihood (ML) detection by searching for the location $m$ which maximized $\Lambda(i, m)$ such as

$$
\bar{I}_{u}(i)=\arg \max _{m}\left[\Lambda_{u}(i, m)\right] .
$$

From this general analysis of the SISO-IR scheme, we derive two theoretical upper bounds on the average bit-error probability according to the type of reception performed (CR or NCR). These bounds will prove valuable to compare the SLIR scheme and the STBC-IR scheme in terms of achievable performances, later in this paper. Each upper bound is related to a $M$-ary orthogonal signal, with a different noise characteristic as described in the following
1) Assumption 1: Considering a peer-to-peer communication, we achieve maximum performances in terms of diversity when a coherent receiver is used, a perfect channel estimation is assumed $\left(\widetilde{\alpha}_{f}=\alpha_{f}, \widetilde{\tau}_{f}=\tau_{f}\right)$, a large-enough value of $N_{f}$ is considered to avoid ISI, and finally there exists $F_{g}$ uncorrelated resolvable multipath components such that $T_{f} \geq F_{g} T_{w}$, $\tau_{f+1}-\tau_{f} \geq T_{w}$, to avoid intra-pulse interferences (IPI). Under these highly theoretical assumptions [14], $\Lambda(i, m)$ can be expressed as

$$
\begin{aligned}
\Lambda(i, m) & =X^{2} \sqrt{\epsilon_{s}} \delta(I(i)-m) \sum_{f=0}^{F_{g}-1} \alpha_{f}^{2} \\
& +X \sqrt{\frac{1}{N_{f}}} \sum_{k=0}^{N_{f}-1} \sum_{f=0}^{F_{g}-1} \alpha_{f} n\left(i, k, \tau_{f}, m\right)
\end{aligned}
$$

where $\epsilon_{s}$ is the transmitted symbol energy. The variance of the total received noise per transmitted symbol, taking into account the matched filter process [15], is given by $\sigma_{s}^{2}=X^{2} N_{0} / 2 \sum_{f=0}^{F_{g}-1} \alpha_{f}^{2}$. According to the symbol-error probability for a $M$-ary orthogonal signal [15]

$$
\begin{gathered}
P_{M}\left(I(i) \rightarrow \bar{I}(i) \mid \alpha_{f}, f=0, \cdots, F_{g}-1\right) \\
\quad<M \prod_{f=0}^{F_{g}-1} \exp \left(-X^{2} \epsilon_{s} \alpha_{f}^{2} / 2 N_{0}\right)
\end{gathered}
$$

In deriving (15), it is assumed that random variables $\alpha_{f}$ are uncorrelated with one another [11], and follow a lognormal $\left(E_{\alpha_{f}}, \sigma_{\alpha}\right)$ distribution. Therefore, the random variable $\alpha_{f}^{2}$ follows a $\log$-normal $\left(2 E_{\alpha_{f}}, 2 \sigma_{\alpha}\right)$ distribution. Averaging (15) with respect to $\alpha_{f}^{2}$, we obtain an upper bound on the average bit-error probability as follows

$$
\begin{gathered}
P_{b}(I(i) \rightarrow \bar{I}(i)) \\
<C\left(\frac{\Gamma(1+K)}{\sqrt{\pi}}\left(\frac{X^{2} \varepsilon_{s}}{2 N_{0}}\left[\prod_{f=0}^{F_{g}-1} e^{2 E_{\alpha_{f}}}\right]^{\frac{1}{F_{g}}}\right)^{-K}\right)^{F_{g}},
\end{gathered}
$$

where $C=M 2^{\left(\log _{2}(M)-1\right)} /\left(2^{\log _{2}(M)}-1\right), K=$ $1 / 2 \sqrt{2} \sigma_{\alpha}$, and $\epsilon_{s} / N_{0}$ is defined as the signal-to-noise ratio (SNR) per symbol. According to (16), a diversity advantage of $K F_{g}$ and a coding advantage of $X^{2} / 2(\Gamma(1+K) / \sqrt{\pi})^{-\frac{1}{K}}\left[\prod_{f=0}^{F_{g}-1} e^{2 E_{\alpha_{f}}}\right]^{\frac{1}{F_{g}}}$ are achieved. A comprehensive derivation from (15) to (16) is provided in the Appendix.

2) Assumption 2: Considering a peer-to-peer communication with resolvable multipath components, applying NCR and assuming a large value for $N_{f}$ to avoid ISI, we approximate the symbol-error probability at high SNR $\left(\epsilon_{s} \gg N_{0}\right)$ by

$$
\begin{gathered}
P_{M}\left(I(i) \rightarrow \bar{I}(i) \mid \alpha_{p, q}, p, q=0, \cdots, P-1, Q-1\right) \\
<M \prod_{p=0}^{P-1} \prod_{q=0}^{Q-1} \exp \left(-X^{2} \epsilon_{s} \alpha_{p, q}^{2} / 4 N_{0}\right)
\end{gathered} .
$$

Finally, averaging (17) with respect to $\alpha_{p, q}^{2}$, we derive an upper bound on the average bit-error probability at high SNR as 


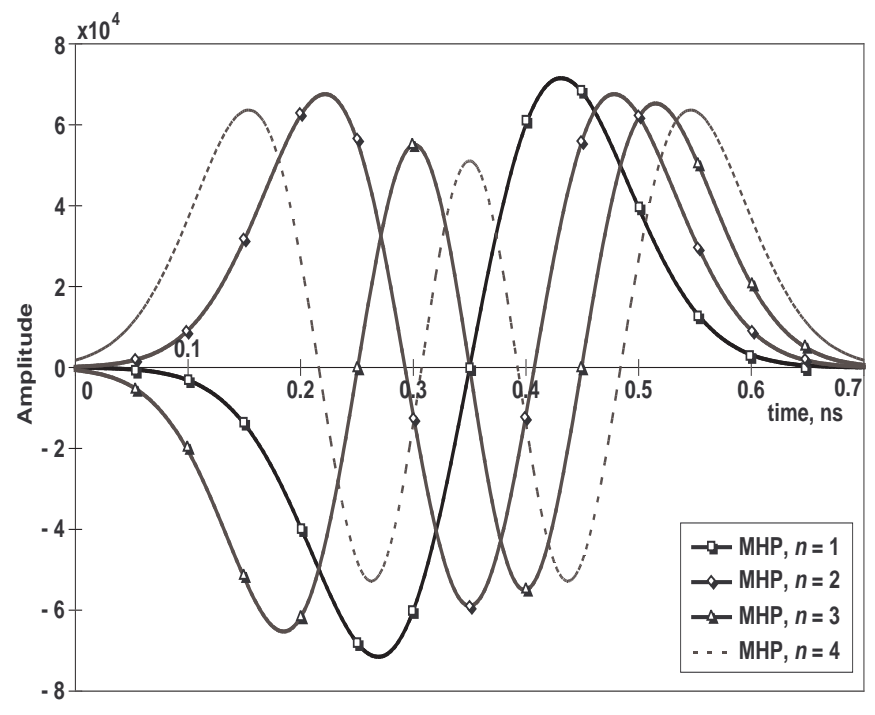

Fig. 1. Time response of normalised to unit-energy MHPs of order $n=1$, $2,3,4$, for $T_{0}=0.2877 \mathrm{~ns}$ and $T_{1}=0.35 \mathrm{~ns}$.

follows

$$
\begin{gathered}
P_{b}(I(i) \rightarrow \bar{I}(i)) \\
<C\left(\frac{\Gamma(1+K)}{\sqrt{\pi}}\left(\frac{X^{2} \epsilon_{s}}{4 N_{0}}\left[\prod_{p=0}^{P-1} \prod_{q=0}^{Q-1} e^{2 E_{\alpha_{p}, q}}\right]^{\frac{1}{P Q}}\right)^{-K}\right)^{P Q} .
\end{gathered}
$$

A diversity advantage of $K P Q$ and a coding gain of $X^{2} / 4(\Gamma(1+K) / \sqrt{\pi})^{-1 / K}\left[\prod_{p=0}^{P-1} \prod_{q=0}^{Q-1} e^{2 E_{\alpha_{p}, q}}\right]^{\frac{1}{P Q}}$ are achieved in this NCR case. Since NCR provides a better achievable diversity advantage, it might provide better performances at higher SNR than CR according to the value of $E_{\alpha_{p, q}}$. Although our bounds in (16) and (18) seem to be highly theoretical as they do not take into account IPI effects which are prominent effects of the channel model implemented here, they are suitable to assess the trend of achievable performances at high SNR, as we show later in Section IV-B.

\section{STBC-IR SCHEME WITH ORTHOGONAL PULSES}

Let us now present our STBC-IR scheme combined with orthogonal pulses. So far, several classes of orthogonal pulses such as MHPs [2], orthogonal pulses based on wavelet packets [16], and orthogonal pulses based on prolate spheroidal wave functions [17] have already been developed. The STBC-IR scheme presented later in this section can be implemented regardless of the type of orthogonal pulses.

\section{A. Modified Hermitian Pulses}

The modified Hermitian set of orthogonal pulses retained our attention owing to the attractive features it provides, and its simplicity of implementation [2]. Each normalised to unitenergy pulse time-domain expression is given by

$$
v_{n}(t)=\frac{\sqrt{2}(-1)^{n} T_{0}^{\left(n-\frac{1}{2}\right)}}{(8 \pi)^{\frac{n}{2}} \sqrt{n !}} e^{2 \pi\left(\frac{t-T_{1}}{T_{0}}\right)^{2}} \frac{d^{n}}{d t^{n}} e^{-4 \pi\left(\frac{t-T_{1}}{T_{0}}\right)^{2}},
$$

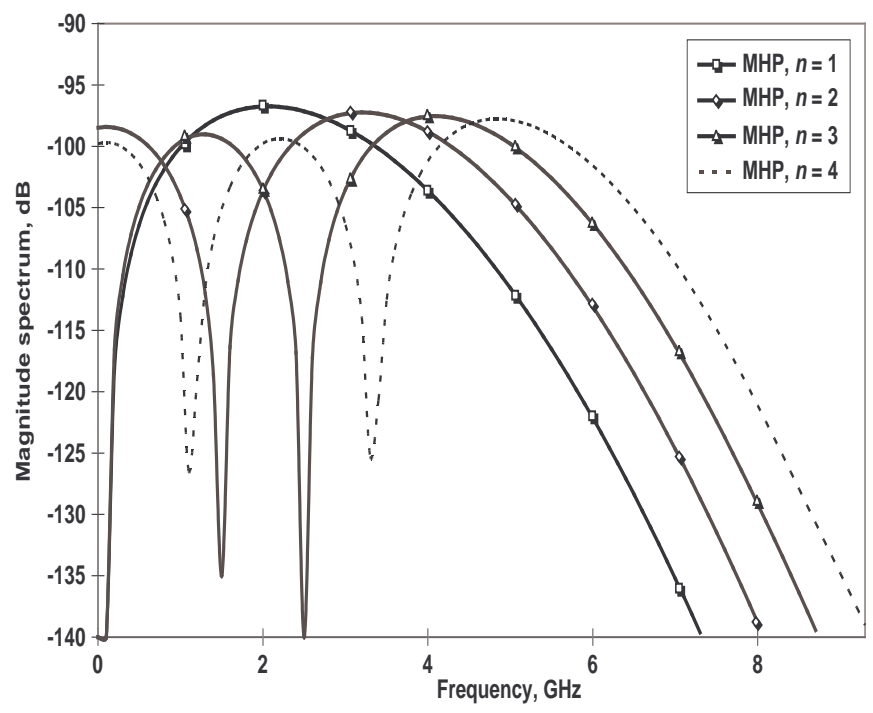

Fig. 2. Magnitude spectrum of normalised to unit-energy MHPs of order $n$ $=1,2,3,4$, for $T_{0}=0.2877 \mathrm{~ns}$ and $T_{1}=0.35 \mathrm{~ns}$.

where $n$ is the order of the pulse, $T_{0}$ defines the width of the pulse and $T_{1}$ determines the center of the pulse. The magnitude spectrum of each normalised to unit-energy MHP is given by

$$
\Upsilon_{n}(f)=\frac{j^{n} F_{0}^{\left(n-\frac{1}{2}\right)}}{(2 \pi)^{\frac{n}{2}} \sqrt{n !}} e^{2 \pi\left(\left(\frac{f}{2 F_{0}}\right)^{2}-j f T_{1}\right)} \frac{d^{n}}{d f^{n}} e^{-\pi\left(\frac{f}{F_{0}}\right)^{2}},
$$

where $F_{0}=1 / T_{0}$. Depicted in Fig. 1 and Fig. 2 are the time response and the magnitude spectrum of normalised to unit-energy' MHPs, for different values of $n$. Otherwise, as reported in [18], the orthogonality between each MHP can be conserved at the receiver side, taking into account distortional antenna effects, via a modification of each MHP shape at the transmitter side.

\section{B. STBC-IR scheme}

The STBC-IR scheme derivation is carried out considering a $N_{t}$ transmit, $N_{\rho}$ receive antenna configuration, a peer-topeer communication, and symbols encoded through a $M$-ary OPPM. The system model chart of our STBC-IR scheme is depicted in Fig. 3. Similar to the SL-IR scheme, bits are first mapped into symbols. Then, the 'symbol stream' is split into $N_{t}$ sub-streams, enabling the transmission of $N_{t}$ different symbols on $N_{t}$ transmit antennas. Next, each symbol substream is shaped into its corresponding waveform $\omega_{n}(t, i)$ via a PPM-encoder and using $N_{t}$ MHP shapers. The transmitted signal sent over the $n$th transmit antenna is expressed as

$$
\begin{aligned}
\omega_{n}(t, i)= & \sqrt{\frac{\epsilon_{s}}{N_{t} N_{f}}} \sum_{l=0}^{N_{t}-1} \sum_{k=0}^{N_{f}-1} v_{c}\left(t-\left([b+I(a)] N_{f}+k\right) T_{w}\right), \\
& \left\{\begin{array}{l}
a=i N_{t}+c, \\
b=\left(i N_{t}+l\right) M, \\
c=\left(N_{t}-l+n\right) \quad \bmod N_{t},
\end{array}\right.
\end{aligned}
$$

where 


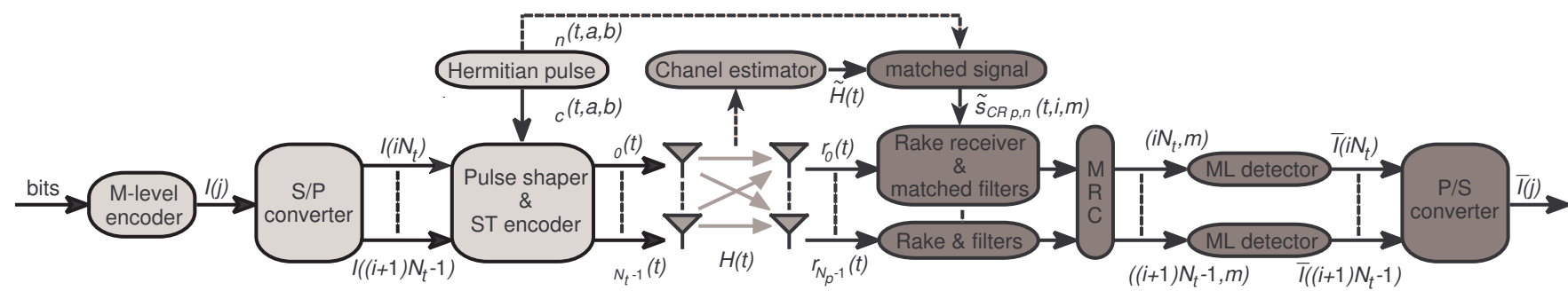

Fig. 3. Block diagram of the $N_{t}$ transmit antennas, $N_{\rho}$ receive antennas, space-time block coding scheme with orthogonal pulses for single band UWB communications.

- $I(a)$ represents the genuine 'symbol stream' before the splitting operation,

- $a$ defines the index of any symbol within the 'symbol stream',

- $b$ determines the position of the waveform representing $I(a)$ within the aggregate signal,

- $c$ is the order of the MHP used to transmit $I(a)$,

- $n \in\left\{0, N_{t}-1\right\}, i \in\left\{0, \frac{N_{s}}{N_{t}}-1\right\}$,

- $\sqrt{\frac{\epsilon_{s}}{N_{f}}}$ is an energy normalization term, and $\frac{1}{\sqrt{N_{t}}}$ guarantees a total transmitted power equal to the one considered in a SL communication system.

Equation (21) describes mathematically how the encoding process is performed for each antenna. It emphasises that encoding is performed at the same time in space and time domains. Moreover, contrarily to a classic block coding scheme implemented in [9], where for most of transmit antenna configurations $\left(N_{t}=3,5,6,7, \ldots\right)$ the length of the block code used has to be greater than $N_{t}$ [5], the length of our block code is always equal to $N_{t}$. For instance, considering $N_{t}=5$, our scheme provides a data rate which is 1.6 times faster than the one provides by a usual block coding scheme.

Each uncorrelated channel link between the $n$th transmit and the $\rho$ th receive antenna is modelled by a finite impulse response signal $h_{\rho, n}(t)$ such as

$$
h_{\rho, n}(t)=X \sum_{p=0}^{P-1} \sum_{q=0}^{Q-1} \alpha_{\rho, n, p, q} \delta\left(t-T_{\rho, n, p}-\tau_{\rho, n, p, q}\right) .
$$

The received signal per receive antenna, per transmitted symbol $r_{\rho}(t, i)$ can then be described by:

$r_{\rho}(t, i)=X \sum_{n=0}^{N_{t}-1} \sum_{p=0}^{P-1} \sum_{q=0}^{Q-1} \alpha_{\rho, n, p, q} \omega_{n}\left(t-T_{\rho, n, p}-\tau_{\rho, n, p, q}, i\right)+n_{\rho}$

where $n_{\rho}(t)$ is a zero-mean gaussian noise with variance $N_{0} / 2$. In order to perform the detection on each transmitted symbol, the same kind of approach applied to the SL-IR scheme is extended. For CR, a DA channel estimation is considered, and the estimated channel impulse response of each possible link $\widetilde{h}_{\rho, n}(t)=\widetilde{X} \sum_{f=0}^{F_{g}-1} \widetilde{\alpha}_{\rho, n, f} \delta\left(t-\widetilde{\tau}_{\rho, n, f}\right)$ is acquired, where $\widetilde{\alpha}_{\rho, n, f}$ is the $f$ th estimated multipath gain of the estimated channel impulse response and $\widetilde{\tau}_{\rho, n, f}$ is the estimated delay of the corresponding multipath gain, regarding the $n$th transmit and $\rho$ th receive antenna. MRC technique allows to combine the energy components of each transmitted symbol sent over different transmit antennas and received over various receive antennas. At the receiver side, $N_{t}$ MHP shapers are used to generate $M \times N_{t}$ waveforms, according to the possible pulse order $n$ and the possible location $m$

$$
\omega_{n}(t, i, l, m)=\sqrt{\frac{1}{N_{t} N_{f}}} \sum_{k=0}^{N_{f}-1} v_{n}\left(t-\left([b+m] N_{f}+k\right) T_{w}\right) .
$$

Then, we form the vector $\Omega_{n}(t, i, m)$ by stacking $\omega_{n}(t, i, l, m)$ as follow

$$
\boldsymbol{\Omega}_{\boldsymbol{n}}(\boldsymbol{t}, \boldsymbol{i}, \boldsymbol{m})=\left[\omega_{n}(t, i, 0, m), \ldots, \omega_{n}\left(t, i, N_{t}-1, m\right)\right] .
$$

In order to implement the effects of block coding, $\widetilde{h}_{\rho, n}(t)$ is re-expressed function of $n$ and $l$ such as

$$
\left.\widetilde{h}_{\rho, n, l}(t)=\widetilde{h}_{\rho,([l+n]} \bmod N_{t}\right)(t) .
$$

Next, we form a channel impulse response vector $\widetilde{\boldsymbol{H}}_{\boldsymbol{\rho}, \boldsymbol{n}}(\boldsymbol{t})$ by stacking $\widetilde{h}_{\rho, n, l}(t)$ as follows

$$
\widetilde{\boldsymbol{H}}_{\boldsymbol{\rho}, \boldsymbol{n}}(\boldsymbol{t})=\left[\widetilde{h}_{\rho, n, 0}(t), \ldots, \widetilde{h}_{\rho, n, l}(t), \ldots, \widetilde{h}_{\rho, n, N_{t}-1}(t)\right],
$$

where $\widetilde{\boldsymbol{H}}_{\boldsymbol{\rho}, \boldsymbol{n}+\boldsymbol{1}}(\boldsymbol{t})$ can be obtained from $\widetilde{\boldsymbol{H}}_{\boldsymbol{\rho}, \boldsymbol{n}}(\boldsymbol{t})$ via a left circular permutation of its elements. Finally, with (25) and (27), an estimated template waveform $s_{\mathrm{CR} \rho, n}(t, i, m)$ is generated as follows

$$
\begin{aligned}
s_{\mathrm{CR} \rho, n}(t, i, m) & =\boldsymbol{\Omega}_{\boldsymbol{n}}(\boldsymbol{t}, \boldsymbol{i}, \boldsymbol{m}) * * \widetilde{\boldsymbol{H}}_{\boldsymbol{\rho}, \boldsymbol{n}}(\boldsymbol{t})^{T} \\
& \left.=\widetilde{X} \sum_{l=0}^{N_{t}-1} \sum_{f=0}^{F_{g}-1} \widetilde{\alpha}_{\rho,([l+n]} \bmod N_{t}\right), f \\
& \left.\times \omega_{n}\left(t-\widetilde{\tau}_{\rho,([l+n]} \bmod N_{t}\right), f, i, l, m\right)
\end{aligned}
$$

$(z y h e r e . *$ stands for the summation of the element-wise convolution of vectors $\Omega_{n}(t, i, m)$ and $\widetilde{H}_{\rho, n}(t)$. Our STBC-IR scheme allows to transmit $N_{t}$ different symbols inside a $N_{t} T_{s}+\widetilde{\tau}_{\max }$ duration, where $\widetilde{\tau}_{\max }=$ $\max \left(\widetilde{\tau}_{\rho,\left([l+n] \bmod N_{t}\right), f}\right)$. So if each signal $s_{\mathrm{CR} \rho, n}(t, i, m)$ is correlated independently with each received signal $r_{\rho}(t, i)$, over $N_{t} T_{s}+\widetilde{\tau}_{\text {max }}$, each symbol is demodulated independently, i.e.

$$
\begin{aligned}
\Lambda(j, m) & =\sum_{\rho=0}^{N_{\rho}-1} \int_{\left(m+i M N_{t}\right) N_{f} T_{w}}^{\left(m+1+\left((i+1) N_{t}-1\right) M\right) N_{f} T_{w}+\widetilde{\tau}_{\max }} r_{\rho}(t, i) \\
& \times s_{\mathrm{CR} \rho, n}(t, i, m) d t
\end{aligned}
$$


where $j=i N_{t}+n$ is the transmitted symbol index of the genuine 'symbol stream'. Considering the assumption in Section II-B1, i.e. all the multipath are resolvable, in the absence of noise the expression of $\Lambda(j, m)$ is given by

$$
\Lambda(j, m)=\frac{X^{2} \sqrt{\varepsilon_{s}}}{N_{t}} \delta(I(j)-m) \sum_{\rho=0}^{N_{\rho}-1} \sum_{v=0}^{N_{t}-1} \sum_{f=0}^{F_{g}-1} \alpha_{\rho, v, f}^{2},
$$

with the variance of the aggregate noise per symbol is such as $\sigma_{s}^{2}=\frac{X^{2} N_{0}}{2 N_{t}} \sum_{\rho=0}^{N_{\rho}-1} \sum_{v=0}^{N_{t}-1} \sum_{f=0}^{F_{g}-1} \alpha_{\rho, v, f}^{2}$. The decision is performed using the ML method, where:

$$
\bar{I}(j)=\arg \max _{m}[\Lambda(j, m)] .
$$

The symbol-error probability under the present assumption is upper bounded by

$$
\begin{gathered}
P_{M}\left(I(j) \rightarrow \bar{I}(j) \mid \alpha_{\rho, v, f}\right) \\
<M \prod_{\rho=0}^{N_{\rho}-1} \prod_{v=0}^{N_{t}-1} \prod_{f=0}^{F_{g}-1} \exp \left(-X^{2} \epsilon_{s} \alpha_{\rho, v, f}^{2} / 2 N_{0} N_{t}\right)
\end{gathered}
$$

Averaging with respect to $\alpha_{\rho, v, f}$, we obtain an upper bound on the average bit-error probability as follows

$$
\begin{gathered}
P_{b}(I(j) \rightarrow \bar{I}(j))<C\left(\frac{\Gamma(1+K)}{\sqrt{\pi}}\right. \\
\left.\times\left(\frac{X^{2} \varepsilon_{s}}{2 N_{0} N_{t}}\left[\prod_{\rho=0}^{N_{\rho}-1} \prod_{v=0}^{N_{t}-1} \prod_{f=0}^{F_{g}-1} e^{2 E_{\alpha_{\rho, v}, f}}\right]^{\frac{1}{N_{\rho} N_{t} F_{g}}}\right)^{-K}\right)^{N_{\rho} N_{t} F_{g}},
\end{gathered}
$$

where $C$ and $K$ are defined in (16). Thus, a diversity advantage of $N_{p} N_{t} K F_{g}$ and a coding advantage of $\frac{X^{2}}{2} N_{t}^{-1}\left(\frac{\Gamma(1+K)}{\sqrt{\pi}}\right)^{-\frac{1}{K}}\left[\prod_{\rho=0}^{N_{\rho}-1} \prod_{v=0}^{N_{t}-1} \prod_{f=0}^{F_{g}-1} e^{2 E_{\alpha_{\rho, v, f}}}\right]^{\frac{1}{N_{\rho} N_{t} F_{g}}}$ are achieved. In Comparison with results obtained in (16) for a SL-IR scheme, given the same data rate, the diversity advantage is clearly increased by a factor $N_{p} N_{t}$ and the coding advantage is decreased by a factor $N_{t}$ and multiplied by $\left[\prod_{\rho=0}^{N_{\rho}-1} \prod_{v=0}^{N_{t}-1} \prod_{f=0}^{F_{g}-1} e^{2 E_{\alpha_{\rho, v}, f}}\right]^{\frac{1}{N_{\rho} N_{t} F_{g}}} /\left[\prod_{f=0}^{F_{g}-1} e^{2 E_{\alpha_{f}}}\right]^{\frac{1}{F_{g}}}$. Next, if we consider NCR, $N_{\rho} N_{t}^{2}$ template waveforms have to be sent prior to each frame transmission. Considering the assumption in Sect. (II-B2), an upper bound on the average bit-error probability, at high $\operatorname{SNR}\left(\epsilon_{s} \gg N_{0}\right)$ is given by

$$
\begin{gathered}
P_{b}(I(j) \rightarrow \bar{I}(j))<C\left(\frac{\Gamma(1+K)}{\sqrt{\pi}}\right. \\
\left.\times\left(\frac{X^{2} \epsilon_{s}}{4 N_{0}}\left[\prod_{\rho=0}^{N_{\rho}-1} \prod_{v=0}^{N_{t}-1} \prod_{p=0}^{P-1} \prod_{q=0}^{Q-1} e^{2 E_{\alpha_{p}, q}}\right]^{\frac{1}{N_{\rho} N_{t} P Q}}\right)^{-K}\right)^{N_{\rho} N_{t} P Q} .
\end{gathered}
$$

Thus, a diversity gain of $N_{\rho} N_{t} K P Q$, and a coding gain of $\frac{X^{2}}{4 N_{t}}\left(\frac{\Gamma(1+K)}{\sqrt{\pi}}\right)^{-\frac{1}{K}}\left[\prod_{\rho=0}^{N_{\rho}-1} \prod_{v=0}^{N_{t}-1} \prod_{p=0}^{P-1} \prod_{q=0}^{Q-1} e^{2 E_{\alpha_{p}, q}}\right]^{\frac{1}{N_{\rho} N_{t} P Q}}$ are achieved in this NCR case. The same kind of behaviour towards the SL-IR scheme is found in terms of decreasing of the coding advantage, and increasing of the diversity advantage by the amount previously stated above for (33).
So far, from the theoretical analysis conducted, (33) and (34) demonstrate that our STBC-IR scheme can achieve transmit and receive diversity. Therefore, depending on the mean value and standard deviation of each multipath component, our STBC-IR scheme might achieve better performances than a SL-IR scheme.

\section{Simulation Parameters And Results}

In this last section, according to simulation parameters, real performances of the STBC-IR scheme over the IEEE channel model are assessed.

\section{A. Simulation Parameters}

Previously introduced in Section II-B, the IEEE UWB channel model has been implemented in this work. Four different scenarios compose this model [11]. Each scenario, CM1 to CM4 is defined accordingly to different channel configurations and characteristics. The CM1 scenario is related to very short distances ( 0 to $4 \mathrm{~m}$ ) and line-of-sight transmissions. The CM2 scenario is defined for the same distances, but with nonline-of-sight (NLOS) antenna configurations. CM3 and CM4 scenarios are defined for NLOS antenna configurations and greater transmission distances, respectively 4 to $10 \mathrm{~m}$, and over $10 \mathrm{~m}$. Moreover, the IEEE UWB channel model assumes a channel impulse response constant over $200 \mu$ s [11], and each of these realisations is statistically independent.

For CR, analysis undertaken in [13] points out the FSrake receiver as a good compromise between performance and complexity. It provides acceptable performance, close to ML one, for much lower complexity. The FS-rake reception technique requires to sample the received signal, after the match filtering process, at least as fast as the Nyquist rate, which involves a sampling rate of several gigahertz. Other assumptions and parameters are as follows:

- The channel estimation (CR case) and synchronization is assumed to be perfect at the receiver side.

- Transmitters and receivers are assumed to be sufficiently apart from each other, thus each link of the channel is considered to be spatially and mutually uncorrelated with the others.

- A 4-OPPM is simulated, with a repetition gain of $10 \mathrm{~dB}$, and a duty cycle of $6 \mathrm{~dB}$.

- Pulse setting: concerning SL-IR scheme simulations, the normalised to unit-energy second derivative of the Gaussian pulse is considered, where

$$
w(t)=\sqrt{\frac{8}{3 T_{0}}}\left(1-4 \pi\left(\frac{t-T_{1}}{T_{0}}\right)^{2}\right) e^{-2 \pi\left(\frac{t-T_{1}}{T_{0}}\right)^{2}} .
$$

As regards for the simulations of the STBC-IR scheme, MHPs of order $n \in\{1,4\}$ are considered. The pulse width of every pulse is set to $0.7 \mathrm{~ns}, T_{0}$ is set to 0.2877 $\mathrm{ns}$, and the pulse center $T_{1}$ is set to $0.35 \mathrm{~ns}$.

- The BER is computed using 400 bits packets, with at least 100 channel realisations of each channel, and 100000 bits transmitted or at least 100 erroneous bits are detected, for each SNR value. Furthermore, as reported in [11], 


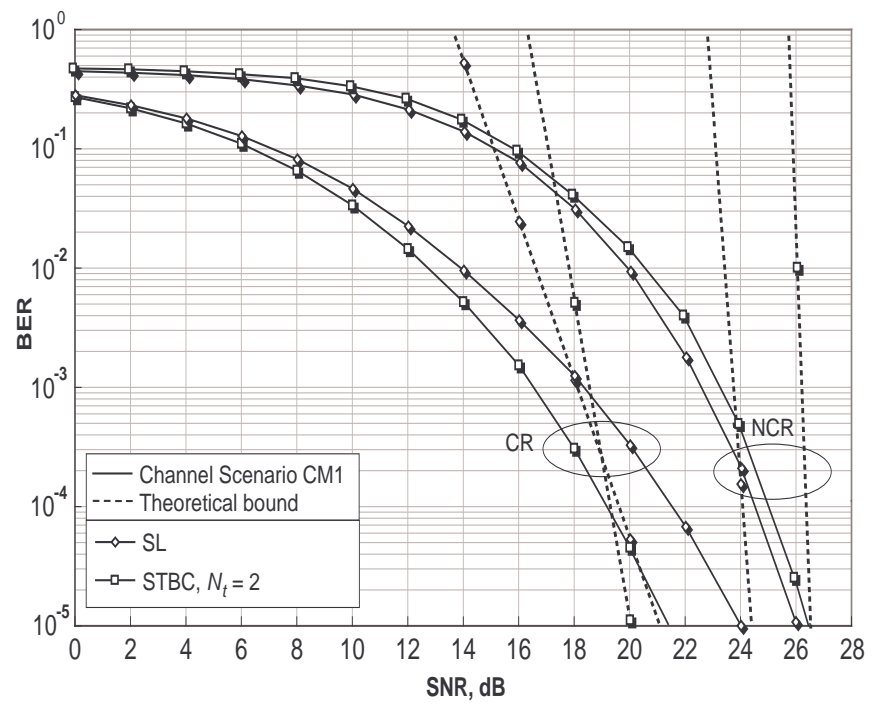

Fig. 4. BER against SNR performances of the SL-IR and STBC-IR schemes, for both NCR and CR $\left(F_{g}=10\right)$, CM1 channel scenario and one receive antenna.

each single dot of a BER against SNR curve represents the average BER, averaged over the best 90 out of 100 channel realisations.

- Both SL-IR scheme and STBC-IR scheme performances are compared for the same data rate.

- Every simulation considered a partial channel impulse response spread over about $18 \mathrm{~ns}$ on average (CM1), about 22 ns on average (CM2), and about 32 ns on average (CM3, CM4).

\section{B. Results}

In Fig. 4, BER against SNR performances of the SLIR scheme and STBC-IR scheme, for both NCR and 10fingers FS-rake $\mathrm{CR}$, are depicted. Here, we consider the CM1 channel scenario and one receive antenna. Moreover, the theoretical bounds derived respectively in equations (16), (18), (33) and (34) are plotted in dotted lines. For NCR, the SL-IR scheme performs slightly better than our STBCIR scheme. This observation is also confirmed by the curves of the theoretical bounds. It means that even if our scheme capture twice the diversity compared to the SL-IR scheme, this diversity advantage remains insufficient compared to the $3 \mathrm{~dB}$ loss, i.e., $10 \log _{10}\left(N_{t}\right)$, and the effects of IPI. Whereas, when applying CR, our STBC-IR scheme performs about 2 $\mathrm{dB}$ better than the SL-IR scheme, at a BER of $10^{-3}$, and the bounds confirm that behaviour for higher SNR. Thus, if the bounds do not account for the IPI, they still provide trend of the performance behaviours at high SNR. Moreover, because the simulation results are averaged over the best 90 out of 100 channel realisations, it is not possible to fairly compare the bounds with the simulation results. Furthermore, as we predicted, NCR provides a better diversity advantage than CR at high SNR. Meanwhile, one can remark that over that kind of channel model, the diversity advantage is less perceptible than over a narrow band indoor channel [9].

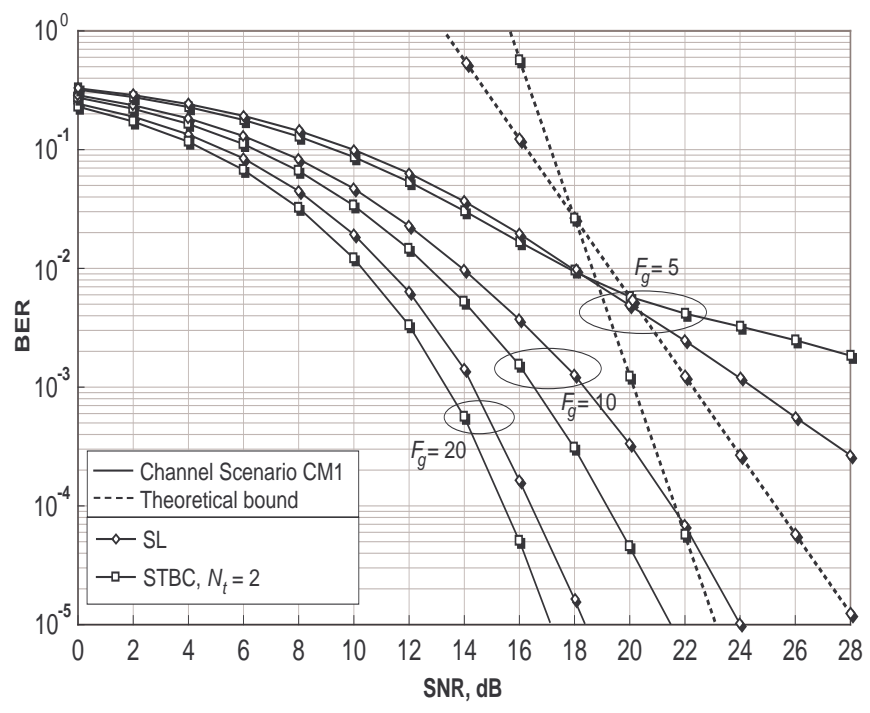

Fig. 5. Effects of the number of FS-Rake fingers on BER against SNR performances, for SL-IR and STBC-IR schemes, CR, $F_{g}=5,10,20, \mathrm{CM} 1$ channel scenario and one receive antenna.

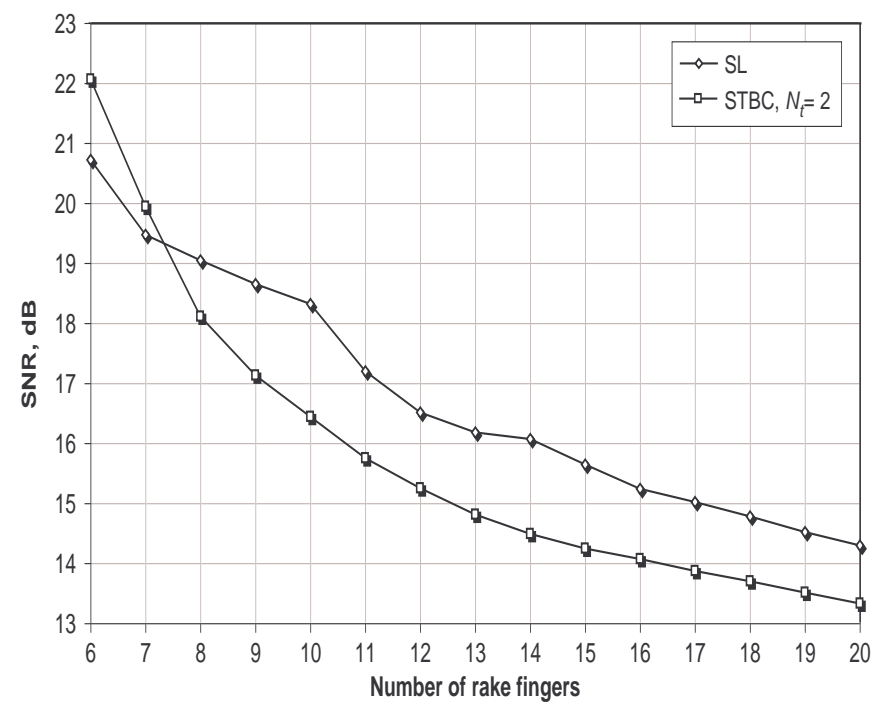

Fig. 6. Effects of the number of FS-rake fingers on the SNR for a fixed BER value of $10^{-3}$, for SL-IR and STBC-IR schemes, CR, CM1 channel scenario and one receive antenna.

Results presented in Fig. 5 assess the effects of the number of FS-rake fingers $F_{g}$ on the system performances, in terms of BER against SNR, for both SL-IR and STBC-IR schemes, considering the CM1 channel scenario and one receive antenna. Results show that for a small number of fingers $\left(F_{g}=5\right)$, our scheme performs worse than the single scheme. However, the theoretical bounds, which are not taking into account the effects of IPI , tell another story. Thus, for a small number of fingers, the diversity gathered by our STBC-IR scheme is not sufficient enough to counter the effects of IPI. Then, when $F_{g}$ increases, the effects of IPI decreases and our scheme, as predicted, gives enhanced performances. Next, Fig. 6 shows the effects of the number of FS-rake fingers $F_{g}$ on the SNR for a fixed BER value of $10^{-3}$. Here, we consider the CM1 


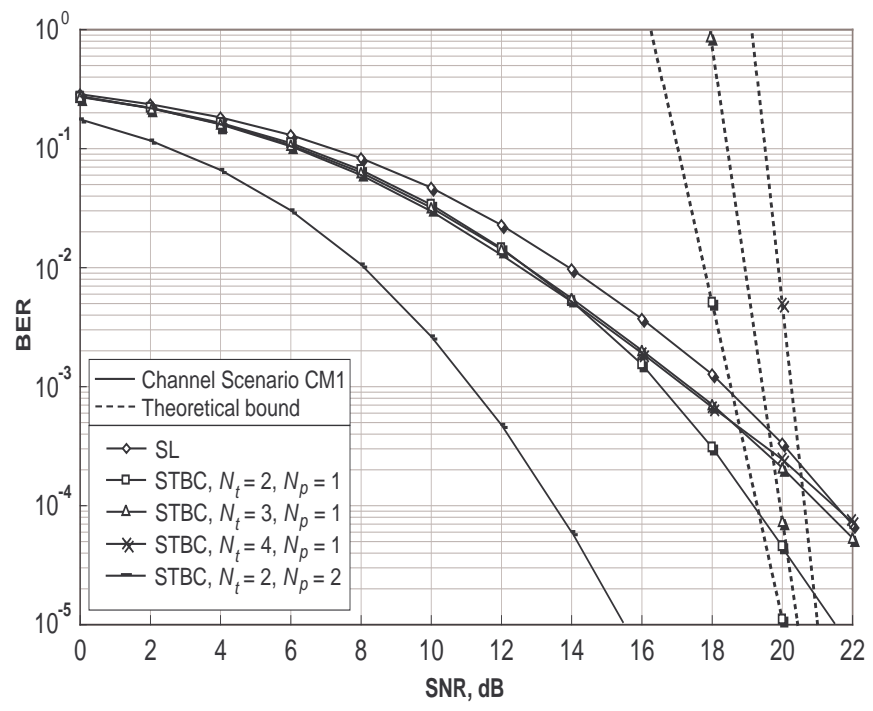

Fig. 7. Effects of the number of transmit and receive antennas on BER against SNR performances, for the STBC-IR scheme, $N_{\rho}=1,2, N_{t}=2,3$, 4, CR, $F_{g}=10$ and CM1 channel scenario.

channel scenario and one receive antenna. It reveals that for small values of $F_{g}, F_{g}<8$, our scheme is outperformed by the SL-IR. For $F_{g}=10$, our scheme produces the maximum possible gain compared to SL-IR, i.e., $2 \mathrm{~dB}$. Between $F_{g}=10$ and $F_{g}=15$, the difference of performances decrease, the SL scheme might be less affected by the IPI than our scheme. Then, for $F_{g}$ values above 15 , the SNR starts to decrease linearly with the number of rake fingers, respectively, 0.18 $\mathrm{dB} /$ fingers for our scheme and $0.23 \mathrm{~dB} /$ fingers for the $\mathrm{SL}$ scheme, which implies a lesser impact of ISI and IPI on the performances. Also, one can remark that a 14-fingers STBC-IR scheme performs as good as a 20 -fingers SL-IR scheme. Therefore, our scheme allows the rake complexity to be reduced.

In Fig. 7, we assess the effects of the number of transmit and receive antennas on the BER against SNR performances regarding the STBC-IR scheme. Here, we consider the CM1 channel scenario, a 10-fingers FS-rake CR and one receive antenna. Surprisingly, increasing the number of transmit antennas decreases the system performances. Thus, the additional diversity provided by the transmit antenna remains insufficient to overcome the $10 \log _{10}\left(N_{t}\right) \mathrm{dB}$ loss which was introduced to insure an equivalent total transmitted power for each scheme. Moreover, adding several channel responses with different time characteristics increases the IPI and partially destroys the orthogonality between pulses. Therefore, our results concerning transmit diversity are dramatically different from those obtained in [9] over a narrow band indoor channel. However, for the receive diversity, the results are quite similar. Therefore, they seem quite flattering compared to the ones obtained for the transmit diversity.

In Fig. 8, the BER against SNR. performances of both schemes are assessed for each channel scenario (CM1 to CM4). Here, we consider a 10-fingers selective FS-rake CR and one receive antenna. Thus, from one scenario to another,

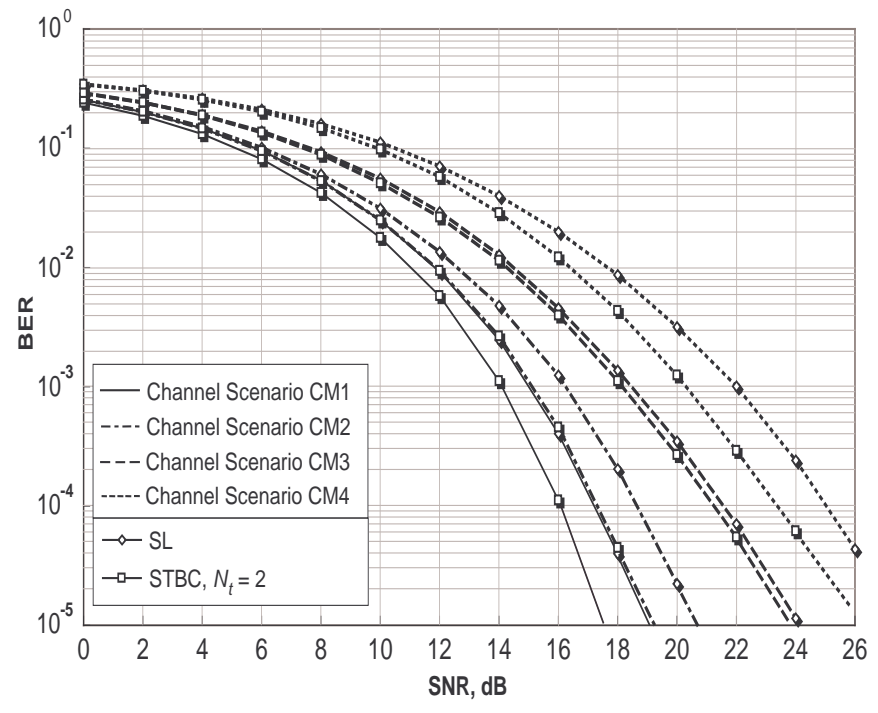

Fig. 8. BER against SNR. performances of the SL-IR and STBC-IR schemes over each different channel scenario (CM1 to CM4), for CR, $F_{g}=10$ and one receive antenna.

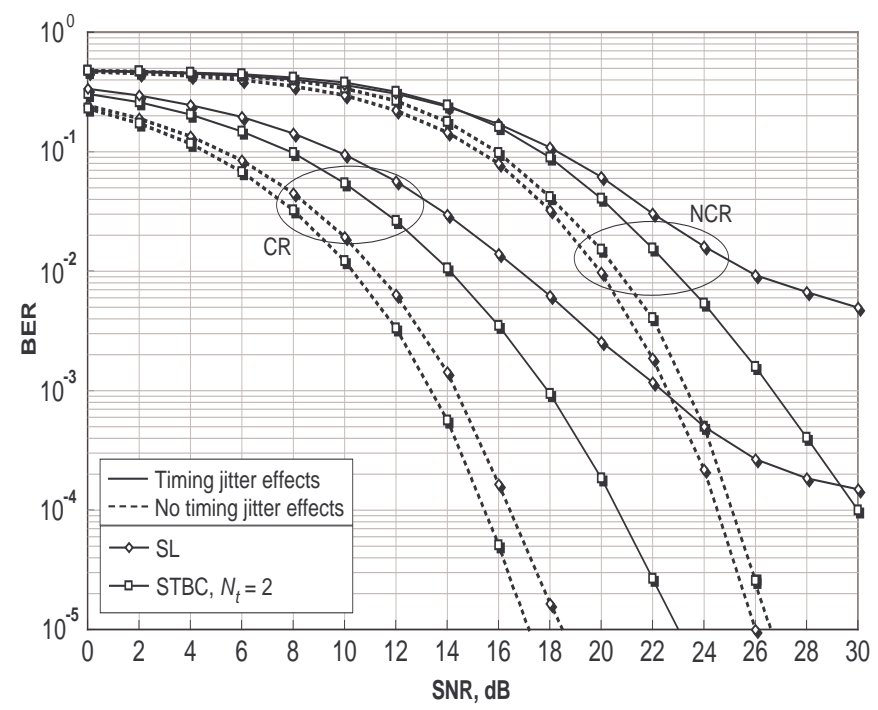

Fig. 9. Effects of the timing jitter on BER against SNR performances, for SL-IR and STBC-IR schemes, both NCR and CR $\left(F_{g}=20\right)$, CM1 channel scenario and one receive antenna.

the performance differences are quite remarkable. Our STBCIR scheme outperformed the SL-IR scheme in every case, and particularly over CM2 and CM4, where the gain at a BER of $10^{-5}$ is about $2 \mathrm{~dB}$. It is also interesting to note than our scheme performed over CM2 as good as the SL-IR over CM1, therefore our scheme could be used to increase the transmission distance.

Finally, in Fig. 9, the main interest of a multiple antenna STBC-IR scheme over that kind of channel model is revealed. The BER against SNR performances of STBC-IR and SL-IR schemes are presented, for both NCR and 20-fingers FS-rake $\mathrm{CR}$ in presence of timing jitter effects and considering one receive antenna. As our results tend to prove, the timing jitter effects have catastrophic consequences on the performances 
of the SL-IR scheme. A performance threshold is reached at high SNR. Alternatively, the spatial diversity provided by our scheme allows us to challenge these effects and to keep acceptable performances at high SNR. The same conclusions were drawn in [9] over a narrow band indoor channel. Therefore, spatial diversity seems to be a suitable way to deal with the timing jitter effects.

\section{CONClusion}

In this paper, we presented and implemented a STBC-IR scheme based on orthogonal pulses for multi-antenna UWB communication systems. Its performances have been assessed over the IEEE UWB channel model [11], applying FS-rake CR or NCR technique. Simplified theoretical bounds have been derived, which seems to describe relatively well the performance trends at high SNR, under certain scenarios. Also, proofs have been given that our STBC-IR scheme can provide both spatial and multipath diversities. The STBC-IR scheme may be used to increase the transmission distance or to reduce the complexity of a rake receiver, keeping similar performances compared to those of the SL-IR scheme. Enhancements seem to be greater for NLOS antenna configurations and very short distances $(0-4 \mathrm{~m})$. Regardless of the number of transmit antennas, the STBC-IR scheme allows to keep the same data rate. It can be implemented regardless of the type of modulation, and class of orthogonal pulses. This work shows the advantage of the receive diversity compared to the transmit diversity over the IEEE UWB channel model. Overall, it also relativises the results found in [9], where the advantages of STBC schemes against SL schemes were obvious over a narrow band indoor channel. Here, the results are less flattering. Nevertheless, STBC schemes remain a strong alternative to combat the destructive effects of timing jitter in UWB communications.

In the future, it will be interesting to investigate the effects of synchronization, antenna coupling, and to test the scheme performances applying realistic estimation techniques reported in [13].

\section{APPENDIX A}

Suppose a random variable $Z$ representing the channel gain follows a log-normal $\left(m_{z}, \sigma_{z}\right)$ probability distribution such as

$$
p(Z)=\frac{1}{Z \sigma_{z} \sqrt{2 \pi}} \exp \left(-\frac{1}{2}\left(\frac{\ln (Z)-m_{z}}{\sigma_{z}}\right)^{2}\right) .
$$

The union bound on the probability of error for any $M$-ary orthogonal signal constellation passing through the channel is expressed as

$$
P_{M}(e)<M \mathrm{Q}\left(\sqrt{S Z^{2}}\right)<M \exp \left(-S Z^{2} / 2\right),
$$

where the previous inequality is based on Chernoff bound, and $S=\epsilon_{s} / N_{0}$ is the SNR. Averaging over $Z$ produces an upper bound on the average bit-error probability as

$$
\begin{gathered}
P_{b}(e)<C \int_{-\infty}^{\infty} \exp \left(-\frac{S}{2} e^{2\left(\sqrt{2} Z \sigma_{z}+m_{z}\right)}\right) \frac{e^{-Z^{2}}}{\sqrt{\pi}} d Z=I, \\
I=C \int_{-\infty}^{\infty} \exp \left(-\frac{S}{2} e^{-2\left(\sqrt{2} Z \sigma_{z}-m_{z}\right)}\right) \frac{e^{-Z^{2}}}{\sqrt{\pi}} d Z=J
\end{gathered}
$$

$$
\begin{gathered}
J \leq C \int_{-\infty}^{\infty} \exp \left(-\frac{S}{2} e^{-2\left(\sqrt{2} Z \sigma_{z}-m_{z}\right)}\right) \frac{e^{-Z}}{\sqrt{\pi}} d Z=L \\
L=C \frac{2^{K} \Gamma(1+K)}{\sqrt{\pi}\left(S e^{2 m_{z}}\right)^{K}}, \\
\Rightarrow P_{b}(e)<C 2^{K} \frac{\Gamma(1+K)}{\sqrt{\pi}}\left(S e^{2 m_{z}}\right)^{-K},
\end{gathered}
$$

where $C=M 2^{\left(\log _{2}(M)-1\right)} /\left(2^{\log _{2}(M)}-1\right)$ and $K=$ $1 / 2 \sqrt{2} \sigma_{z}$.

\section{ACKNOWLEDGMENT}

The authors wish to thank Naveel Riaz for his helpful comments in improving the quality of this paper.

\section{REFERENCES}

[1] Scholtz, R. A.: 'Multiple access with time-hopping impulse radio', in Proc. Milcom, Boston, USA, Oct. 1993, pp. 447-450

[2] Ghavami, M., Michael, L., and Kohno, R.: 'Ultra Wideband Signals and Systems in Communication Engineering' (Wiley Europe, May 2004)

[3] Win, M. Z., and Scholtz, R. A.: 'Ultra-wide bandwidth time-hopping spread-spectrum impulse radio for wireless multiple access communications', IEEE Trans. Commun., Apr. 2000, 48, (4), pp. 679-691

[4] Alamouti, S.: 'A simple transmit diversity technique for wireless communications', IEEE J. Select. Areas Commun., Oct. 2000, 16, (8), pp. 1451-1458

[5] Tarokh, V., Jafarkhani, H., and Calderbank, A. R.: 'Space-time block codes from orthogonal designs', IEEE Trans. Inform. Theory, July 1999, 45, (5), pp. 1456-1467

[6] Tarokh, V., Seshadri, N., and Calderbank, A. R.: 'Space-time codes for high data rate wireless communications: Performance criterion and code construction', IEEE Trans. Inform. Theory, Mar. 1998, 44, (2), pp. 17441765

[7] Foschini, G. J.: 'Layered space-time architecture for wireless communication in a fading environment when using multiple antennas', Bell Labs Technical Journal, Nov. 1996, 1, (2), pp. 41-59

[8] Yang, L., and Giannakis, G. B.: 'Space-time coding for impulse radio', in Proc. IEEE UWB Systems, Baltimore, USA, May 2002, pp. 235-240,

[9] Yang, L., and Giannakis, G. B.: 'Analog space-time coding for multiantenna ultra-wideband transmissions', IEEE Trans. Commun., Mar. 2004, 52, (3), pp. 507-517

[10] Heliot, F., Ghavami, M., and Nakhai, M. R.: 'Space-time block coding with orthogonal pulses for impulse radio', in Proc. WPMC, Oct. 2003, Yokosuka, Japan, 2, pp. 517-521

[11] Foerster J., and et al.: 'Channel modeling sub-committee report final', IEEE P802.15 Wireless Personal Area Networks, Tech. Rep. P802.1502/490r1-SG3a, Feb. 2003.

[12] Saleh, A. A. M., and Valenzuela, R. A.: 'A statistical model for indoor multipath propagation', IEEE J. Select. Areas Commun., Feb. 1987., 5, (2), pp. 128-137

[13] Mielczarek, B., Wessman, M.-O., and Svensson, A.: 'Performance of coherent uwb rake receivers using different channel estimators', in Proc. IWUWBS, June 2003, Oulu, Finland

[14] Liu, H.: 'Error performance of a pulse amplitude and position modulated ultra-wideband system over lognormal fading channel', IEEE Commun. Lett., Nov. 2003, 7, (11), pp. 531-533

[15] Proakis, J.: 'Digital Communications' (4th ed. McGraw-Hill, Feb. 2001)

[16] Ciolino, S., Ghavami, M., and Aghvami, H.: 'UWB pulse shape modulation system using wavelet packets', in Proc. IWUWBS, June 2003, Oulu, Finland

[17] Dilmaghani, R., Ghavami, M., Allen, B., and Aghvami, H.: 'Novel pulse shaping using prolate spheroidal wave functions for uwb', in Proc. PIMRC, Sept. 2003, Beijing, China

[18] Abreu, G. T. F. de, Mitchell, C., and Kohno, R.: 'On the orthogonality of hermite pulses for ultra wideband communications systems', in Proc. WPMC, Oct. 2003, Yokosuka, Japan, 2, pp. 288-291 